\title{
Safe Walking in VR
}

\author{
Maurício Sousa \\ INESC-ID Lisboa, IST, ULisboa \\ antonio.sousa@tecnico.ulisboa.pt
}

\author{
Daniel Mendes \\ INESC-ID Lisboa, IST, ULisboa \\ danielmendes@tecnico.ulisboa.pt
}

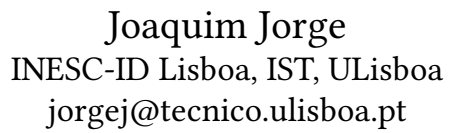

Joaquim Jorge

jorgej@tecnico.ulisboa.pt

\begin{abstract}
Common natural walking techniques for navigating in virtual environments feature constraints that make it difficult to use those methods in cramped home environments. Indeed, natural walking requires unobstructed and open space, to allow users to roam around without fear of stumbling on obstacles while immersed in a virtual world. In this work, we propose a new virtual locomotion technique, CWIP-AVR, that allows people to take advantage of the available physical space and empowers them to use natural walking to navigate in the virtual world. To inform users about real world hazards our approach uses augmented virtual reality visual indicators. A user evaluation suggests that CWIP-AVR allows people to navigate safely, while switching between locomotion modes flexibly and maintaining a adequate degree of immersion.
\end{abstract}

\section{CCS CONCEPTS}

- Human-centered computing $\rightarrow$ Computer supported cooperative work; Mixed / augmented reality.

\section{KEYWORDS}

Locomotion, Walking in Place, Augmented Virtual Reality

\section{ACM Reference Format:}

Maurício Sousa, Daniel Mendes, and Joaquim Jorge. 2019. Safe Walking in VR. In The 17th International Conference on Virtual-Reality Continuum and its Applications in Industry (VRCAI '19), November 14-16, 2019, Brisbane, QLD, Australia. ACM, New York, NY, USA, 2 pages. https://doi.org/10.1145/ 3359997.3365737

\section{INTRODUCTION}

Navigation is one of the most common and important tasks in virtual environments. Natural Walking (NW) is useful for withinreach displacements, especially circular movements around small objects of interest, in that it replicates the real world movement into the virtual world in that a person's steps map into their avatar in the VE. Walking in Place (WIP) [Usoh et al. 1999] is more suitable for far-reaching locomotion that cannot be readily accommodated within a small physical space, in that it induces virtual forward movement by simulating steps while marching in the same place in the real world. In this paper, we introduce Combined Walking in Place (CWIP), which combines NW with WIP with seamless transitions between the two. Though this technique people can access places in the VE that lie beyond the confines of the physical

Permission to make digital or hard copies of part or all of this work for personal or classroom use is granted without fee provided that copies are not made or distributed for profit or commercial advantage and that copies bear this notice and the full citation on the first page. Copyrights for third-party components of this work must be honored

For all other uses, contact the owner/author(s).

VRCAI '19, November 14-16, 2019, Brisbane, QLD, Australia

(C) 2019 Copyright held by the owner/author(s).

ACM ISBN 978-1-4503-7002-8/19/11.

https://doi.org/10.1145/3359997.3365737

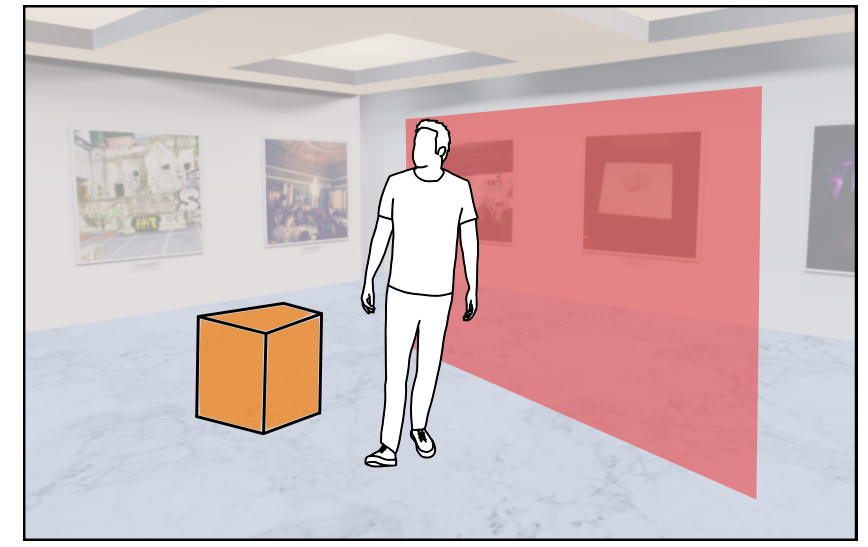

Figure 1: To achieve safe walking in VR, our approach combines Walking-In-Place locomotion technique with Natural Walking and virtual proximity indicators for obstacles and room boundaries.

room available via WIP. They can naturally switch to $N W$ when the point of interest in the VE is within the range of the physical space available.

However, using $N W$ while immersed becomes a recipe for disaster, as people become blissfully unaware of their physical surroundings. To tackle this, we developed a warning system that uses visual indicators to inform the user of the existence and location of physical obstacles, such as the limits of the available area and other encumbrances, as depicted in Figure 1. Additionally, to increase people awareness of those obstacles we also implemented visual and audio signals to direct their attention to the augmented visual indicators. We call our combined approach CWIP-AVR, resulting from combining CWIP navigation with Augmented Virtual Reality to implement collision warnings.

\section{CWIP-AVR}

Our approach combines Walking in Place with Natural Walking in novel manners to achieve more flexible and powerful ways to support locomotion within domestic settings. Furthermore, our approach adopts Augmented Virtual Reality to make it possible to roam VEs safely within the confines of household dwellings.

\subsection{CWIP - Combined Walking in Place}

CWIP allows people to seamlessly and automatically alternate between two different locomotion techniques in the virtual world. To this end, we determine which of the two techniques should be used at a given time according to the current state of a CWIP State Machine, according to three possible states: Stationary, NW or WIP. 
The state transitions are determined by: (1) whether the person is in motion; (2) if not in motion, whether they are simulating steps.

If the system determines that the user is in motion the current state is set to NW, and the movement of the avatar in the virtual world directly corresponds to their physical motion. If the person is not walking and is not marching in place, the system is in the Stationary state. In this state the avatar is at rest in virtual world, in a fashion similar to the behavior of the NW state, in that the avatar follows the locomotion / immobility of the person. If the user is not moving but is simulating steps, the system should be in the WIP state. In this state, the movement of the avatar in the VE is determined by the Walking in Place locomotion technique.

\subsection{AVR - Augmented Virtual Reality}

This component is responsible for informing the person about the physical space limits and obstacles in their path,during their navigation in the virtual world. To accomplish this, we are augmenting the Virtual World representation using three distinct elements: Visual Indicators, Warning Arrows and Sound Alerts.

The Visual Indicators adds synthetic objects to the virtual world, that behave independently of it and have a distinct visual appearance. They serve to signal the location of hazards and obstacles to progression in the real world (Figure 2). These indicators can be divided into two Groups: limits are represented by translucent planes that depict the physical limits of the available space in front that the person; obstacles, rendered as solid parallelepipeds, indicate the location of obstacles in the real world, such as chairs, tables, beds and other pieces of furniture, that lie in the path of the user. Indicators' color and transparency depend on their distance to the users. These distances are split into four zones: Normal Zone $(>1.2 \mathrm{~m})$ no indicator; Pre-Warning Zone $(<1.2 \mathrm{~m})$ - white with maximum

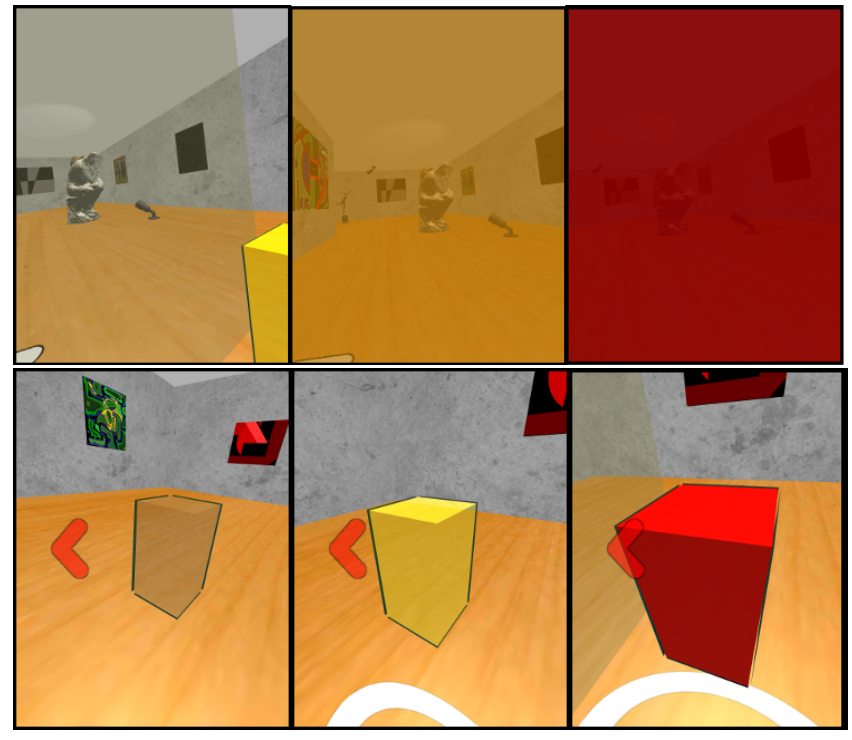

Figure 2: Rendering a Visual Indicator of a limit in different zones (top: limits, bottom: objects): Pre-Warning (left), Warning (middle), and Danger (right). The red Warning Arrow indicates another obstacle to the left of the user transparency; Warning Zone $(<0.8 \mathrm{~m})$ - yellow; and Danger Zone $(<$ $0.4 \mathrm{~m})$ - red and almost opaque. Changes between zones are made with a linear progression.

To inform users of real-world hazards outside their field of view, we included two types of feedback that direct the user's attention to the Visual Indicators: Warning Arrow are red arrows depicted on the side of the FoV to indicate the direction of obstacles nearby in the virtual world but out of the person's sight; Sound Alerts are audible alarms that warn the user that they are about to enter the Danger Zone of an obstacle outside their field of view. These alarms can stop in one of two situations: the person leaves the Danger Zone of the hazard, or they look directly at the corresponding Visual Indicator, thus showing that they are aware of the existence of the obstacle.

\section{EVALUATION}

To validate our approach we carried out a user study $(N=20)$. The study consisted of a series of three navigation tasks in two different conditions: CWIP-AVR, and a baseline. The baseline is an adaptation of Steam VR's Chaperone system, that allows people to observe the physical work throw a camera feed. To execute the tasks, participants were asked to navigate in a VE, featuring a virtual art gallery twenty meters long up to a 90 degree curve followed by another twenty-meter long gallery leading to a podium with an object on top (finish line). We implemented the evaluation prototype using a Samsung GearVR headset and employed five Microsoft Kinect v2 with the Creepy Tracker Toolkit [Sousa et al. 2017] for tracking. In regards to our walking in place approach, we adapted the Bruno et al. [Bruno et al. 2017] work.

All participants were able to master CWIP with little effort. This establishes our approach as an effective way to navigate virtual environments using commodity depth sensors. Although results indicate that the baseline is more efficient and the best to communicate the position of the person in the real world, participants reported that it broke the sense of presence. Additionally, the baseline resulted in a considerably greater number of exits from the designated interaction area (baseline: 13 participants, CWIP-AVR: 3 participants). These resulted in collisions with the boundaries of the interaction area, that suggests that the baseline approach is unsafe. Indeed, each exit could have resulted in the participant hitting a wall if there were no safety distance between the limits of the interaction area and the physical lab walls.

\section{ACKNOWLEDGMENTS}

This work was supported by FCT (ref. UID/CEC/50021/2013).

\section{REFERENCES}

Luís Bruno, Maurício Sousa, Alfredo Ferreira, João Madeiras Pereira, and Joaquim Jorge. 2017. Hip-directed walking-in-place using a single depth camera. International fournal of Human-Computer Studies 105 (2017), 1-11.

Maurício Sousa, Daniel Mendes, Rafael Kuffner Dos Anjos, Daniel Medeiros, Alfredo Ferreira, Alberto Raposo, João Madeiras Pereira, and Joaquim Jorge. 2017. Creepy tracker toolkit for context-aware interfaces. In Proceedings of the 2017 ACM International Conference on Interactive Surfaces and Spaces. ACM, 191-200.

Martin Usoh, Kevin Arthur, Mary Whitton C., Rui Bastos, Anthony Steed, Mel Slater, and Frederick Brooks P. 1999. Walking $>$ Walking-in-Place > Flying, in Virtual Environments. SIGGRAPH' 99 Proceedings of the 26th annual conference on Computer graphics and interactive techniques (1999), 359-364. https://doi.org/10.1145/311535. 311589 